\title{
Identifying appropriate sampling and modelling approaches for analysing distributional patterns of Antarctic terrestrial arthropods along the Victoria Land latitudinal gradient
}

\author{
TANCREDI CARUSO ${ }^{1}$, IAN D. HOGG ${ }^{2}$ and ROBERTO BARGAGLI ${ }^{3}$ \\ ${ }^{1}$ Institute of Biology, Dahlem Center of Plant Sciences, Freie Universität Berlin, Altensteinstraße 6, 14195 Berlin, Germany \\ ${ }^{2}$ Department of Biological Sciences, University of Waikato, Private Bag 3105, Hamilton 3240, New Zealand \\ ${ }^{3}$ Department of Environmental Sciences "G. Sarfatti”, University of Siena, via P.A. Mattioli, 4, 53100 Siena, Italy \\ tancredi.caruso@fu-berlin.de
}

\begin{abstract}
Biotic communities in Antarctic terrestrial ecosystems are relatively simple and often lack higher trophic levels (e.g. predators); thus, it is often assumed that species' distributions are mainly affected by abiotic factors such as climatic conditions, which change with increasing latitude, altitude and/or distance from the coast. However, it is becoming increasingly apparent that factors other than geographical gradients affect the distribution of organisms with low dispersal capability such as the terrestrial arthropods. In Victoria Land (East Antarctica) the distribution of springtail (Collembola) and mite (Acari) species vary at scales that range from a few square centimetres to regional and continental. Different species show different scales of variation that relate to factors such as local geological and glaciological history, and biotic interactions, but only weakly with latitudinal/altitudinal gradients. Here, we review the relevant literature and outline more appropriate sampling designs as well as suitable modelling techniques (e.g. linear mixed models and eigenvector mapping), that will more adequately address and identify the range of factors responsible for the distribution of terrestrial arthropods in Antarctica.
\end{abstract}

Received 12 August 2009, accepted 1 May 2010

Key words: Antarctica, mites, modelling designs, phylogeography, sampling, spatial distribution, springtails

\section{Introduction}

Much of the biogeographical and ecological research of Antarctic terrestrial organisms has assumed that abiotic factors are primarily responsible for explaining their distributions (e.g. Convey et al. 2003, Hogg et al. 2006). Generally, environmental stress increases with increasing latitude, altitude or the distance from coastal oases and it has been assumed that these geographical gradients play an important role in structuring the spatial distributions of species (Adams et al. 2006, Sinclair \& Stevens 2006, Chown \& Convey 2006, 2007). However, based on earlier investigations (e.g. Janetschek 1967, Usher \& Booth 1986), it is also known that the effects of geographical clines (climate) on structuring the distribution patterns of several Antarctic organisms (including arthropods) are mediated by several local-scale processes that may result in patchy and discontinuous distributions. For example, Adams et al. (2006) note that the non-overlapping distributions of endemic arthropods in Victoria Land and the Trans-Antarctic Mountains $\left(60-86^{\circ} \mathrm{S}\right)$ is indicative of historical isolation. Peat et al. (2007) performed an extensive evaluation of the flora in the same regions and concluded that there was no evidence for a latitudinal cline in this area, even though a cline was clearly evident in the Antarctic Peninsula. Peat et al. (2007) also suggested that spatial patterns in lichen species indicate vicariant distribution and that most mosses are recent colonists.

Although increasing latitude is often linked to the progressive loss of species and functional groups (especially among the macrofauna and macroflora), it is not always clear whether the latitudinal decrease in diversity is the result of a harsher climate or if it simply reflects different Antarctic biogeographical zones. For example, in the maritime Antarctic $\left(60-72^{\circ} \mathrm{S}\right)$, Lawley et al. (2004) found the highest eukaryotic diversity at the southern limit and interpreted this to be a consequence of closer proximity to ice free areas during recent glacial cycles. Several environmental and historical processes may also interact at a hierarchy of levels and scales, making it challenging to detect and interpret spatial patterns such as those due to the latitude (see Chown \& Convey 2006 for review).

Here, we focus on latitudinal patterns of terrestrial arthropod distribution within Victoria Land and the Trans-Antarctic Mountains (Fig. 1). The distribution of taxa such as springtails (Collembola) and mites (Acari) appears independent of latitudinal gradients; in fact, some 'hotspot' regions occur as far south as the Scott Glacier $\left(86^{\circ} 30^{\prime}\right.$ 'S; Broady \& Weinstein 1998). Species' numbers increase slightly towards Cape Hallett in the north (up to $70^{\circ} \mathrm{S}$ ) but not in a linear fashion (Strandtmann 1967, Wise 1967, 1971). Indeed, the 


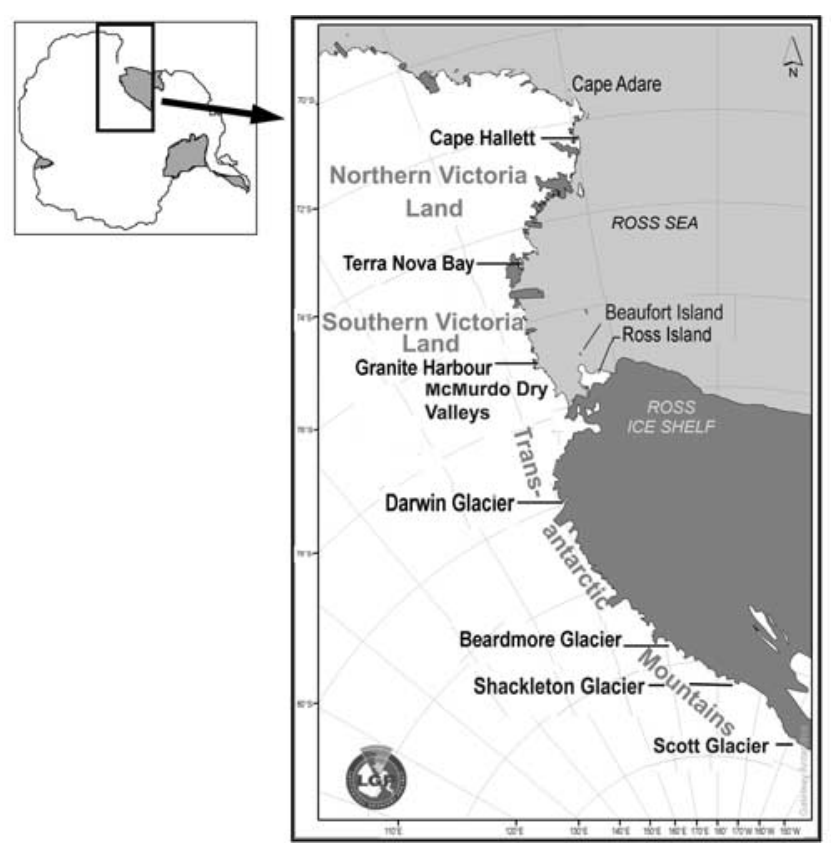

Fig. 1. Location of Victoria Land latitudinal gradient (inset) showing place names referred to in the text.

phylogeography of Antarctic terrestrial arthropods is likely to have a more complex explanation (e.g. McGaughran et al. 2010). These taxa show a high degree of endemism and their distributions seem mostly influenced by the presence of refugia from which recolonization may have occurred as terrestrial habitats became accessible (e.g. following glacial maxima). Thus, local geological and glaciological events may be more important than latitudinally-driven climatic conditions in determining the distribution of springtails and mites (Wise 1967, Brundin 1970, Stevens et al. 2006; see also reviews by Convey \& Stevens 2007, Convey et al. 2008, 2009). On this basis, we review these recent data and re-evaluate the spatial distributions of terrestrial arthropod taxa along the Victoria Land latitudinal gradient. We then present appropriate theoretical sampling designs and modelling frameworks that will more adequately account for the varying scales of spatial distributions.

\section{Species' distributions at local and micro-geographical scales}

Usher \& Booth (1986) found that the distributions of springtails and mites in the maritime Antarctic change with spatial scale, from a few centimetres to metres, and that different species differed in the scales at which they were distributed. For example, the predatory mite Gamasellus sp. was randomly distributed over a range of spatial scales, while springtails in the genus Cryptopygus were clumped at scales of 10 to $60 \mathrm{~cm}$. These results were corroborated by two recent surveys of arthropod community structure in northern Victoria Land (Caruso \& Bargagli 2007, Caruso et al. 2007), where mites and springtails had population densities that were significantly different on scales of square centimetres to square metres and that different species had different scales of variations. Furthermore, no strong relationships were found between physical environmental parameters and the distribution of the springtail Gressittacantha terranova Wise (Caruso et al. 2007). Similar patterns were also observed at Cape Hallett by Sinclair et al. (2006). However, earlier workers such as Janetschek (1967) had predicted that optimal conditions of temperature and moisture determined the presence of arthropods. Instead, geographic sampling coordinates were one of the most important predictors of species' occurrence (Sinclair et al. 2006), indicating the presence of spatial autocorrelation (sensu Legendre \& Legendre 1998). Although Janetschek's (1967) prediction is sound in terms of general ecological principles, the detection of spurious relationships between environmental variables and species distributions may be challenging when organisms exhibit multiple scales of variability in their distributions (e.g. Usher \& Booth 1986, Sinclair et al. 2006), and imply high levels of autocorrelation. Spatial autocorrelation causes samples in close proximity to be more similar to each other than samples further apart. For soil arthropods, these correlations mainly depend on dispersal ability, conspecific attraction and population fluctuations, which can vary on a local scale of a few centimetres (e.g. Berthet 1964, Ojala \& Huhta 2001, Pugh 2003, Hawes et al. 2007, 2008). A high degree of autocorrelation in a species' localscale distribution is usually interpreted as strong evidence of within-population drivers (e.g. Legendre 1993, Legendre \& Legendre 1998, Dormann et al. 2007), even though it may also arise from missing relevant environmental or biological factors that act at scales that are not resolved by the sampling design (i.e. in terms of the size of sampling unit and the mean distance among sampling units). For example, in the case of arthropods from northern Victoria Land, the fine scale of variation and available evidence (e.g. correlation with soil pigments Sinclair 2001, Caruso et al. 2007), suggest that biotic interactions related to food source (algae) may influence arthropod distributions. Therefore, if the distribution of a food source is spatially structured at scales smaller than the scale at which other factors such as moisture may affect consumers, and if the food source is not accounted for explicitly, the residuals from an analysis relating moisture to consumer distribution will be spatially autocorrelated because of the dependency on food.

Although the occurrence of spatial autocorrelation is not surprising, it highlights some critical methodological and sampling issues that need to be addressed by current ecological studies of Antarctic terrestrial invertebrates. Most relevant modelling techniques and statistical tests assume independence of error terms, an assumption clearly violated by autocorrelation which would also invalidate 
conclusions based on classical statistics (Legendre 1993, Dormann et al. 2007). This may be only partly resolved by hierarchical nested sampling designs and the use of linear mixed models, as tentatively suggested by Caruso \& Bargagli (2007). If the scale of spatial patterning is well known it is probable that the use of nested designs and random components in linear mixed models will be effective. However, if some degree of autocorrelation remains within error terms, it is necessary to use appropriate modelling approaches that can explicitly account for error correlation (Dormann et al. 2007). Furthermore, even when using genetics for tracking the relationships among populations, it may still be difficult to detect the main scales of spatial patterning in Antarctic species such as Desoria klovstadi (Carpenter) (Stevens et al. 2007). While this may seem problematic, we believe it actually provides a new opportunity for the biological interpretation of patchily-distributed Antarctic arthropods and addresses the multiple spatial scales at which species' distributions can vary. Enhanced knowledge of these critical scales of variation will allow for a more effective interpretation of the processes responsible for the observed patterns.

In order to develop appropriate sampling strategies, we can start with an example of a model taken from Caruso et al. (2007) which related the probability of occurrence $(P)$ for the mite Stereotydeus belli (Trouessart) relative to soil water content, texture and sampling date. Five coastal ice free areas in northern Victoria Land were studied. At each of the five sites there were six plots and these were sampled on two occasions for a total of 60 observations. The effect of the three variables on $P$ was found to be independent of sampling site. These results were influenced by the scale of the sampling sites and the general aim for which the data were collected (i.e. the influence of environmental variables on species occurrence). However, assume we had collected several more samples on a single date with sampling plots of $10 \mathrm{~m}^{2}$ nested within each of the five sites of $500 \mathrm{~m}^{2}$. Simplifying the original linear model of Caruso et al. (2007) to consider only moisture as an example and ignoring possible interactions, $P$ can be related to its drivers according to

$$
P=\text { Water }+ \text { Site, }
$$

while a possible linear mixed model related to the hypothetical nested sampling strategy would be

$$
P=\text { Water }+ \text { Site }+(\text { random }: \text { Site } \mid \text { Plot }),
$$

where the last term (in parentheses) accounts for random processes at scales within site (Laird \& Ware 1982, Davidian \& Giltinan 1995, Pinheiro \& Bates 2000). The symbol "|" indicates that Plot is randomly repeated within Site and will affect to some extent the probability of occurrence within each site. According to this model, sites are fixed and the factor Site defines the largest spatial scale (i.e. the five coastal ice free areas). The factor Plot consists

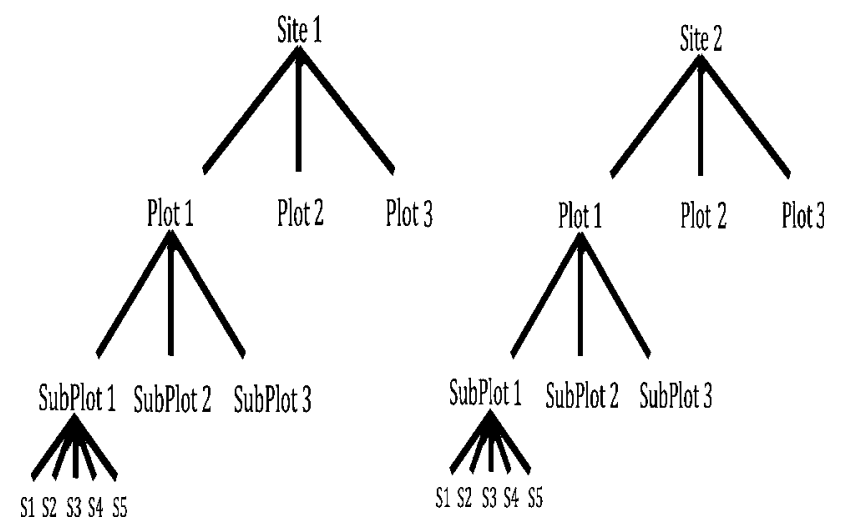

Fig. 2. A hypothetical sampling design with Plots and Subplots randomly nested within the fixed factor Site. In this example, within each subplot there are five randomly collected samples or units of observation (S1-S5).

of several random levels (the various sampling plots) that are randomly nested within each Site. Plots within a site that differ in the probability of occurrence $(P)$ will result in a larger error for the estimate of mean $P$ expected within each site. In the absence of significant variability within each site (i.e. among plots), different sites may have significantly differing basal levels of $P$. In this case, physical parameters of each sampling unit (e.g. soil water content) may increase (or decrease) the mean $P$ within a site (Caruso et al. 2007).

For example, assume the mean probability $P( \pm$ s.e. $)$ of finding a target species beneath a randomly collected stone was $0.4 \pm 0.05$ at one site and $0.2 \pm 0.04$ at a second site. Here, the model of Eq. (1) would work and also account for the effect of drivers like soil water content. However, if within each site $P$ varies at a scale of a few square metres, detecting a significant difference between sites could be enhanced as a consequence of a too narrow estimate of the error (i.e. the smaller error will result in a higher probability of detecting a significant difference) and would not account for biologically relevant smaller scale variations. At the same time, using a nested sampling design and analysing data according to the model of Eq. (1), would probably produce autocorrelation and inflated significant differences between sites (or alternatively, in the case of high variability result in the missing of differences). Using the hierarchically nested plots within each site and linear mixed models of Eq. (2) would account for such effects and thus allow unbiased comparison at the scale of Sites.

Most published papers on Antarctic arthropods have assumed a model similar to Eq. (1) (e.g. Sinclair 2001, Sinclair et al. 2006, Caruso et al. 2007), while on the basis of more recent research (Dormann et al. 2007, Caruso \& Bargagli 2007), and considering multiple scale patterns observed by earlier workers (e.g. Usher \& Booth 1986), we suggest that Eq. (2) is likely to be better, at least in some 
cases. In order to consider other scales of variation, it is possible to improve the nested design with the addition of other hierarchical levels, for instance subplot within plot according to

$$
P=\text { Water }+ \text { Site }+(\text { random }: \text { Site } \mid \text { Plot } / \text { Subplot })
$$

where the random component now accounts not only for plots randomly nested within sites but also for subplots randomly nested within plot (“/”). A graphical representation of this sampling scheme showing two sampling sites with nested plots is shown in Fig. 2. Site is a fixed factor and represents the main natural units under comparison (e.g. two sites along a latitudinal gradient). The spatial scale that separates the two sites, as well as the size of the sites, represents the largest and main scale in the analysis. However, there can be factors acting at several smaller scales that could potentially bias the comparison of the two sites. In order to deal with these sources of variability, randomly located Plots and Subplots (smaller plots within the larger plots) can be included in the sampling design. Within the smallest plots (here Subplots) several repeated observations or samples (S1 to S5) are randomly collected in the field and constitute the observational units of the study. In a classical nested design, the samples repeated within subplots are assumed to be independent. If this is not true because of spatial autocorrelation (i.e. the closer the samples are the more similar they are), mixed models with autocorrelation functions can be used to allow for a spatially unbiased data analysis.

The most complex scenario would be spatial autocorrelation within the nested levels, i.e. Plot and Subplot. However, if the definition of the hierarchical scales was based on sound knowledge of a species' spatial distributions and/or on ground-truthed hypotheses of the main biotic and abiotic drivers, it is possible that the inclusion of random effects in the linear mixed models would account for most of the residual variations. If not, there are appropriate methods that can specify the structure of autocorrelation required within the nested levels of the mixed models (e.g. Dormann et al. 2007). Our ability to understand the relative role of biotic and abiotic parameters on the distribution of arthropods will be possible only if we account for these patterns.

In the absence of a hierarchically nested sampling design (e.g. Sinclair et al. 2006, Caruso et al. 2007), and if significant spatial autocorrelation exists, Eq. (1) can be modified to take this into account. For example, one possibility is the autocovariate model,

$$
P=\text { Water }+ \text { Site }+\rho \mathbf{A},
$$

where in the last term $\rho$ is the coefficient of the autocovariate matrix $\mathbf{A}$, which estimates how much the response variable at any one sampling point reflects response values at surrounding points (Augustin et al. 1996, He et al. 2003, Dormann et al. 2007). In the case of directional autocorrelation, multiple autocovariates may be considered. For example, if there are predominantly two directions of autocorrelations, (e.g. north-south and east-west), the model becomes

$$
P=\text { Water }+ \text { Site }+\rho \mathbf{A}_{\text {north-south }}+\rho \mathbf{A}_{\text {west-east }}
$$

Directional autocorrelation appears to be present at Cape Hallett, in northern Victoria Land as the spatial proximity of samples (measured by their geographic distance), rather than environmental correlates such as soil water, was a better if not the best predictor of community composition (Sinclair et al. 2006).

Recently, techniques based on the principle of eigenvector mapping have been developed to unravel the complexity of spatial patterns over the range of scales that the sampling design encompasses (Legendre et al. 2005, Dray et al. 2006). We believe that these techniques may significantly improve the spatial analysis of the distribution of Antarctic arthropods, given that their distribution is characterized by multiple scales of variability and that this, in turn, is likely to violate assumptions made by linear mixed models and autologistic regressions. The proposed techniques use principal coordinate analysis (PCoA) for deriving the eigenvectors of a sample distance matrix weighted by some appropriate measure of geographic proximity. The above spatial distance matrix is viewed as made by the product of two matrices: 1) a connectivity matrix, which is a binary matrix coding for a network linking samples in a connected graph, with links among samples representing routes of interactions (neighbourhoods), and 2) a weighting matrix, which is based on functions describing the similarity among samples (in the case of spatial analysis, similarity depends on geographic distance. However, similarity may also be based on other measures such as genetic distances. By varying the two matrices (i.e. the routes by which samples are more likely to interact and the intensity of the interactions as a function of distance), many spatial patterns can be accounted for. Each of the eigenvectors extracted from the weighted spatial matrix through PCoA accounts for one of the possible patterns of autocorrelation, which in terms of multiple scales range from broad to fine. For individual studies, the actual size of "broad" and "fine" depends on the scales accounted for by the sampling design. The set of eigenvectors that best accounts for the multiple spatial-scale patterns observed is then used as a predictor of the response variable (i.e., species density or occurrence, community alpha and beta diversity or others). The set allows one to determine the source of variance in the response variable in terms of pure spatial variables (mapping eigenvectors), spatially structured environmental variables, and pure environmental variables. Thus, the general models would look like

$$
P=\text { Water }+E M s
$$

where $E M s$ is a linear combination of eigenvectors that best account for spatial patterns. Technical aspects for constructing a connectivity matrix are provided by Dray et al. (2006). 
Here, we highlight the flexibility of the technique in that it allows one to account for multiple spatial patterns such as those observed in earlier studies (e.g. Usher \& Booth 1986). This means that it is possible to test hypotheses on the structure of autocorrelation, which may provide crucial insights on the processes that drive population distribution. Furthermore, the eigenvector mapping techniques do not require a particular sampling strategy and may fit systematic sampling based on regular grids as well as irregular sampling based on randomising the location of samples. The only critical point is that the scale accounted for by the sampling must fit the scale of the processes to be investigated in terms of: 1) the size of sampling unit (i.e. it should be smaller than the process to be detected), 2) the distance among sampling units (i.e. smaller than the geographical distances by which investigated processes spatially structure the distribution of the response variables), and 3) the extent of the study area (i.e. comparable to the geographical range encompassed by the processes under investigation).

The relevance of micro-geographical and multiple scale variability is demonstrated by recent studies on the population genetics of the springtails Gomphiocephalus hodgsoni, G. terranova and Desoria klovstadi as well as the prostigmatid mite Stereotydeus mollis (e.g. Stevens et al. 2007, McGaughran et al. 2008, 2010). On the basis of molecular analyses it has been suggested that springtail and mite populations are remnants of much larger ancient ranges and that recolonization has occurred from glacial refuges since the Last Glacial Maximum (c. 17000 years ago; Stevens \& Hogg 2003, McGaughran et al. 2008, 2010). These data also indicate minimal or no present-day gene flow among populations of the same putative species, sometimes separated at very local scales. This suggests that small populations have their own history, are patchily distributed, and that colonization events are rare and stochastic. Because populations are also interacting with their environment, such multiple scale patterns within a species or even a population must be addressed in ecological studies that aim to model and interpret the spatial distribution of taxa.

\section{Species' distributions at larger scales}

Populations of the springtail Gomphiocephalus terranova in northern Victoria Land are known to cluster into three geographical regions divided by glacial barriers, which block both dispersal and gene flow along latitudinal trajectories (Fanciulli et al. 2001). Accordingly, there is evidence of latitudinal gradients for some species. Recent biogeographical and molecular evidence has highlighted the potential role of historical events in determining species' distributions (e.g. McGaughran et al. 2010), and latitudinal spatial patterns could still result from these events. In some cases, these events can date back to the Miocene. This may potentially challenge our current thinking which is usually based on large-scale geoglaciological models (e.g. see Convey et al. 2008, 2009 for reviews). Unfortunately, such models do not account for the infinitesimally small fraction $(<0.4 \%)$ of the landscape which would have remained ice free during glacial maxima and where animal survival would have occurred through geological time scales (Convey \& Stevens 2007, Convey et al. 2008, 2009). Under this scenario, Victoria Land is particularly difficult to interpret. Here, climatic spatial clines are mainly related to latitude, altitude and distance from the sea (Chown \& Convey 2006). However, if a series of historical events (e.g. glaciations) have structured species' phylogeographies, it may be difficult to unravel the climatic components that may also be driving the observed spatial patterns.

Caruso et al. (2009) tested whether the distributions of the Victoria Land springtails G. terranova, G. hodgsoni and Friesea grisea (Schaëffer) could be modelled as a function of latitude, longitude, altitude and distance from the sea. A database was constructed using presence/absence records of these species from surveys performed in 133 sites during the last ten years (1996-2006) along the entire latitudinal span of Victoria Land. No general spatial patterns were apparent for the three species. Instead, the three species clearly showed their own specific spatial structures. For instance, longitude was more important than latitude in explaining the distribution of $F$. grisea and G. terranova. In contrast, the distribution of G. hodgsoni was correlated with latitude and included a quadratic term (unimodal pattern) of latitude while neither longitude nor squared longitude was supported. For both $F$. grisea and $G$. terranova the most plausible models also included a quadratic term for longitude and a linear term for latitude. So, while previous evidence suggested that the distribution of these springtail species was influenced predominantly by latitude (Sinclair \& Stevens 2006), quantitative statistical analyses do not support this conclusion. When integrated with data on the phylogeography and physiology of the species (Sinclair \& Sjursen 2001), it is more likely that historical and environmental components have interacted and structured the observed latitudinal patterns for species along the Victoria Land gradient. Studies performed at a smaller scale also support this view (e.g. Stevens et al. 2007).

While climatic spatial clines in Victoria Land are mainly related to latitude, longitude may also include environmental components that are relevant to those of latitude or altitude. Thus, longitudinal autocorrelation patterns, rather than the seemingly more relevant latitudinal components, suggest that non-environmental drivers are also responsible for structuring species' distributions. This remains true despite latitude and/or altitude being key variables in correlative models and their association with latitudinal shifts in species' physiologies (e.g. Sinclair \& Sjursen 2001).

On the basis of available data it can be hypothesized that longitudinal, as well as part of the latitudinal, variation in the species' distributions is driven by historical, geo-glaciological 
events that have shaped the species' contemporary geographical ranges in Victoria Land (e.g. Chown \& Convey 2006). In particular, sites that had/have a relatively higher probability of being colonized can equally be past refuges or sites nearer to older glacial refuges that may have been subjected to a higher probability of immigrants. While the actual mechanisms of colonization and survival are generally not known (but see Hawes et al. 2008), it is clear that a population that has colonized or survived at a site following glaciation must be considered environmentally well adapted. It is separated from other populations of the same species and thus contributes to large-scale patterns of the species from only a local ecological and evolutionary perspective. Accordingly, the idiosyncrasies of processes at local and larger scales in Antarctica are the result of interactions between historical and environmental drivers and their resulting effect on terrestrial arthropod distribution.

\section{Conclusions}

Previous research on the distribution of Antarctic invertebrates has been influenced by the assumption that abiotic factors and especially geographical ranges are responsible for most of the variance observed in species' distributions, because environmental stress increases with increasing latitude or altitude. However, for taxa with low dispersal capabilities, such as the terrestrial arthropods, complex, multiple spatial-scale patterns have been observed and available ecological data combined with more recent molecular analyses suggest that: 1) species vary in their distributions at scales that range from a few square centimetres to regional levels, 2) different species show different scales of variation, 3) spatial-scale variability is likely to depend on different processes at different scales, and 4) at larger scales (regional levels and above), the spatial distributions of arthropod taxa are only weakly correlated with latitude. Recent phylogeographic research strongly supports the hypothesis that geo-glaciological events and the presence of past refuges are more important than latitudinal variations in climatic and environmental conditions in determining the large-scale distributions of Antarctic arthropod taxa. We conclude that the role of biotic interactions has been largely underestimated and that more appropriate sampling designs (e.g. hierarchically nested and randomized sampling), as well as modelling approaches (e.g. linear mixed model including functions of autocorrelation, eigenvector mapping) are required to properly evaluate the spatial distributions of Antarctic arthropod taxa.

\section{Acknowledgements}

This study was supported by the Italian PNRA (Programma Nazionale di Ricerche in Antartide), the New Zealand Foundation for Research Science and Technology (UOWX0710) and Antarctica New Zealand (events K020/ 24/28). It contributes to the EBESA IPY project no. 452 , the Latitudinal Gradient Project (LGP), and the SCAR-EBA (Evolution and Biodiversity in the Antarctic) Programme. We are grateful to Mark Stevens and two anonymous reviewers for their constructive comments which substantially improved the manuscript.

\section{References}

Adams, B.J., Bardgett, R.D., Ayres, E., Wall, D.H., Aislabie, J., Bamforth, S., Bargagli, R., Cary, C., Cavacini, P., Connell, L., Convey, P., Fell, J.W., Frati, F., Hogg, I.D., Newsham, K.K., O’Donnell, A., Russell, N., Seppelt, R.D. \& Stevens, M.I. 2006. Diversity and distribution of Victoria Land biota. Soil Biology and Biochemistry, 38, 3003-3018.

Augustin, N.H.M., Mugglestone, A. \& Buckland, S.T. 1996. An autologistic model for the spatial distribution of wildlife. Journal of Applied Ecology, 33, 339-347.

Berthet, P.L. 1964. Field study of the mobility of oribatei (Acari) using radioactive tagging. Journal of Animal Ecology, 33, 443-449.

Broady, P.A. \& Weinstein, R.N. 1998. Algae, lichens and fungi in La Gorce Mountains, Antarctica. Antarctic Science, 10, 376-385.

Brundin, L. 1970. Antarctic land faunas and their history. In Holdgate, M.W., ed. Antarctic ecology, vol. 1. London: Academic Press, 41-53.

Caruso, T. \& Bargagli, R. 2007. Assessing abundance and diversity patterns of soil microarthropod assemblages in northern Victoria Land (Antarctica). Polar Biology, 30, 895-902.

Caruso, T., Borghini, F., Bucci, C., Colacevich, A. \& Bargagli, R. 2007. Modelling local-scale determinants and the probability of microarthropod species occurrence in Antarctic soils. Soil Biology and Biochemistry, 39, 2949-2956.

Caruso, T., Hogg, i.D., Carapelli, A., Frati, F. \& Bargagli, R. 2009. Large-scale spatial patterns in the distribution of Collembola (Hexapoda) species in Antarctic terrestrial ecosystems. Journal of Biogeography, 36, 879-886.

Chown, S.L. \& Convey, P. 2006. Biogeography. In Bergstom, D.M., Convey, P. \& Huiskes, H.L., eds. Trends in Antarctic terrestrial and limnetic ecosystems: Antarctica as a global indicator. Dordrecht: Springer, 55-70.

Chown, S.L. \& Convey, P. 2007. Spatial and temporal variability across life's hierarchies in the terrestrial Antarctic. Philosophical Transactions of Royal Society, B362, 2307-2331.

Convey, P. \& Stevens, M.I. 2007. Antarctic biodiversity. Science, 317, $1877-1878$.

Convey, P., Block, W. \& Peat, H.J. 2003. Soil arthropods as indicators of water stress in Antarctic terrestrial habitats? Global Change Biology, 9, $1718-1730$.

Convey, P., Gibson, J.E.A., Hillenbrand, C.-D., Hodgson, D.A., Pugh, P.J.A., Smellie, J.L. \& Stevens, M.I. 2008. Antarctic terrestrial life - challenging the history of the frozen continent? Biological Reviews, 83, 103-117.

Convey, P., Stevens, M.I., Hodgson, D.A., Smellie, J.L., Hillenbrand, C.-D., Barnes, D.K.A., Clarke, A., Pugh, P.J.A., Linse, K. \& Cary, S.C. 2009. Exploring biological constraints on the glacial history of Antarctica. Quaternary Science Reviews, 28, 3035-3048.

Davidian, M. \& Giltinan, D.M. 1995. Nonlinear mixed effects models for repeated measurement data. New York: Chapman \& Hall, 360 pp.

Dormann, C.F., McPherson, J.M., Araújo, M.B., Bivand, R., Bolliger, J., Carl, G., Davies, R.G., Hirzel, A., Jetz, W., Kissling, W.D., Kühn, I., Ohlemüller, R., Peres-Neto, P.R., Reineking, B., Schröder, B., SChURR, F.M. \& WiLSON, R. 2007. Methods to account for spatial autocorrelation in the analysis of species distributional data: a review. Ecography, 30, 609-628.

Dray, S., Legendre, P. \& Peres-Neto, P.R. 2006. Spatial modelling: a comprehensive framework for principal coordinate analysis of neighbour matrices (PCNM). Ecological Modelling, 196, 483-493. 
Fanciulli, P., Summa, D., Dallai, R. \& Frati, F. 2001. High levels of genetic variability and population differentiation in Gressittacantha terranova (Collembola, Hexapoda) from Victoria Land, Antarctica. Antarctic Science, 13, 246-254.

Hawes, T.C., Worland, M.R., Bale, J.S. \& Convey, P. 2008. Rafting in Antarctic Collembola. Journal of Zoology, 274, 44-50.

Hawes, T.C., Worland, M.R., Convey, P. \& Bale, J.S. 2007. Aerial dispersal of springtails on the Antarctic Peninsula: implications for local distribution and demography. Antarctic Science, 19, 3-10.

He, F.L., Zноu, J. \& Zнu, H. 2003. Autologistic regression model for the distribution of vegetation. Journal of Agricultural, Biological and Environmental Statistics, 8, 205-222.

HogG, I.D., Cary, S.C., Convey, P., Newsham, K.K., O’Donnell, A.G., Adams, B.J., Aislabie, J., Frati, F., Stevens, M.I. \& Wall, D.H. 2006. Biotic interactions in Antarctic terrestrial ecosystems: are they a factor? Soil Biology \& Biochemistry, 38, 3035-3040.

JANETSCHEK, H. 1967. Arthropod ecology of south Victoria Land. Antarctic Research Series, 10, 205-293.

LAIRD, N.M. \& WARE, J.H. 1982. Random-effects models for longitudinal data. Biometrics, 38, 963-974.

Lawley, B., Ripley, S., Bridge, P. \& Convey, P. 2004. Molecular analysis of geographic patterns of eukaryotic diversity in Antarctic soils. Applied Environmental Microbiology, 70, 5963-5972.

LEGENDRE, P. 1993. Spatial autocorrelation: trouble or new paradigm? Ecology, 74, 1659-1673.

Legendre, P. \& Legendre, L. 1998. Numerical ecology, 2nd ed. Amsterdam: Elsevier Science, 853 pp.

Legendre, P., Borcard, D. \& Peres-Neto, P.R. 2005. Analyzing beta diversity: partitioning the spatial variation of community composition data. Ecological Monographs, 75, 435-450.

McGaughran, A., Hogg, I.D. \& Stevens, M.I. 2008. Patterns of population genetic structure for springtails and mites in southern Victoria Land, Antarctica. Molecular Phylogenetics and Evolution, 46, 606-618.

McGaughran, A., Torricelli, G., Carapelli, A., Frati, F., Stevens, M.I., Convey, P. \& HogG, I.D. 2010. Contrasting phylogeographic patterns for springtails reflect different evolutionary histories between the Antarctic Peninsula and continental Antarctica. Journal of Biogeography, 37, 103-119.
Ojala, R. \& Huhta, V. 2001. Dispersal of microarthropods in forest soil. Pedobiologia, 45, 443-450.

Peat, H.J., Clarke, A. \& Convey, P. 2007. Diversity and biogeography of the Antarctic flora. Journal of Biogeography, 34, 132-146.

Pinheiro, J.C. \& Bates, D.M. 2000. Mixed-effects models in S and S-plus. Berlin: Springer, $530 \mathrm{pp}$.

Pugh, P.J.A. 2003. Have mites (Acarina: Arachnida) colonised Antarctica and the islands of the Southern Ocean via air currents? Polar Record, 39, 239-244.

SincLAIR, B.J. 2001. On the distribution of terrestrial invertebrates at Cape Bird, Ross Island, Antarctica. Polar Biology, 24, 394-400.

Sinclair, B.J. \& SuURSEN, H. 2001. Cold tolerance of the Antarctic springtail Gomphiocephalus hodgsoni (Collembola: Hypogastruridae). Antarctic Science, 13, 271-279.

Sinclair, B.J. \& Stevens, M.I. 2006. Terrestrial microarthropods of Victoria Land and Queen Maud Mountains, Antarctica: implications of climate changes. Soil Biology and Biochemistry, 38, 3158-3170.

Sinclair, B.J., Scott, M.B., Klok, C.J., Terblanche, J.S., Marshall, D.J., Reyers, B. \& Chown, S.L. 2006. Determinants of terrestrial arthropod community at Cape Hallett, Antarctica. Antarctic Science, 18, 303-312.

Stevens, M.I. \& HogG, I.D. 2003. Long-term isolation and recent range expansion revealed for the endemic springtail Gomphiocephalus hodgsoni from southern Victoria Land, Antarctica. Molecular Ecology, 12, 2357-2369.

Stevens, M.I., Greenslade, P., Hogg, I.D. \& Sunnucks, P. 2006. Southern Hemisphere springtails: could any have survived glaciation of Antarctica? Molecular Biology and Evolution, 23, 874-882.

Stevens, M.I., Frati, F., McGaughran, A., Sinsanti, G. \& Hogg, I.D. 2007. Phylogeographic structure suggests multiple glacial refugia in northern Victoria Land for the endemic Antarctic springtail Desoria klovstadi (Collembola, Isotomidae). Zoologica Scripta, 36, 201-212.

Strandtmann, R.W. 1967. Terrestrial Prostigmata (Trombidiform mites). Antarctic Research Series, 10, 51-80.

Usher, M.B. \& Bоотн, R.G. 1986. Arthropod communities in a maritime Antarctic moss-turf habitat: multiple scales of pattern in the mites and Collembola. Journal of Animal Ecology, 55, 155-170.

Wise, K.A.J. 1967. Collembola (springtails). Antarctic Research Series, 10, 123-148.

Wise, K.A.J. 1971. The Collembola of Antarctica. Pacific Insects Monograph, 25, 57-74. 